\title{
A CHARACTERIZATION OF CLOSED 3-MANIFOLDS WITH SPINES CONTAINING NO WILD ARCS
}

\author{
GERHARD W. KNUTSON ${ }^{1}$
}

In this paper we shall prove that a closed 3-manifold has a spine that contains no wild arc if and only if it is a connected sum of closed 3-manifolds each of which is $S^{3}, S^{1} \times S^{2}, R P^{3}$ (real projective 3-space) or $J$ (the twisted $S^{2}$ bundle over $S^{1}$ ).

Since all closed 3-manifolds have a combinatorial triangulation [3], we will work in the combinatorial category. A subcomplex $A$ of a closed 3-manifold $M$ is a spine of $M$ if $A$ has a regular neighborhood of which the closure of the complement is a closed 3-ball. The connected sum of two closed 3-manifolds $M \# M^{\prime}$ is obtained by removing the interior of closed 3-ball from each manifold and matching the boundaries by means of a homeomorphism (or an orientation reversing homeomorphism if both manifolds are orientable). The disk sum of two compact 3-manifolds with boundary $N \triangle N^{\prime}$ is obtained by pasting a 2-ball of $\mathrm{Bd} N$ onto a 2-ball of $\mathrm{Bd} N^{\prime}$.

Lemma 1. If $A$ and $A^{\prime}$ are spines of closed 3-manifolds $M$ and $M^{\prime}$, $M \# M^{\prime}$ has a spine homeomorphic to the one-point union of $A$ and $A^{\prime}$ (written $A \bigvee A^{\prime}$ ).

Proof. If we pick the 3-balls of the connected sum to be 3-simplexes of some triangulation that meet the respective spines, the lemma follows.

Since $R P^{3}$ has $R P^{2}$ as a spine and both $S^{1} \times S^{2}$ and $J$ have $S^{1} \bigvee S^{2}$ as spines, Lemma 1 establishes the sufficiency of the main theorem.

For the rest of this paper we will assume that $A$ is a spine of the closed 3-manifold $M$ such that $A$ does not collapse onto any proper subset of itself and $A$ contains no wild arc. A 3-book $B$ in $M$ is defined to be the union of 3 closed disks in $M$ such that each pair of disks meet precisely on a single arc on the boundary of each. By [4], $A$ contains no 3-book. Let $\operatorname{Lk}(x, X)$ and $\operatorname{St}(x, X)$ be the link and star of $x$ in a second derived subdivision of a complex triangulating $X$. If $a$ is a vertex of $A, \operatorname{Lk}(a, A)$ is the disjoint union of $p 1$-spheres and $q$ points. Such a vertex will be called a $(p, q)$-point. We establish a series of elementary moves that will transform $A$ into a spine of $M$ that is a

Received by the editors July $10,1968$.

1 This paper is part of the author's doctoral dissertation at Michigan State University, prepared under the supervision of P. H. Doyle, while the author was supported by an NSF Traineeship. 
one-point union of 1-spheres and 2-manifolds. Notice that it will not be possible to fix a triangulation of $M$, and we will subdivide whenever it is necessary.

Move A: Let $a$ be a $(p, q)$-point of $A$ with $p q>2$. Let $x$ be an isolated point of $\operatorname{Lk}(a, A)$. In $\operatorname{Lk}(a, M)$ there is an arc $C$ with $\mathrm{Bd} C$ $=A \cap C=x \cup y$, where $y$ is a point of a 1-sphere of $\operatorname{Lk}(a, A)$. There is a 2-cell $B$ in $\operatorname{St}(a, M)$ with $A \cap B=A \cap B d B=a * x \cup a * y$ and Bd $B$ $=a * x \cup a * y \cup C$. Here "*" denotes the join operator. An $A$-move expands $A$ to $A \cup B$ and collapses from $a * x$ across $B$ onto $\mathrm{Cl}(A-a * x) \cup C$.

Move B: Let $a$ be a $(p, 0)$-point of $A$ with $p>1$. Then there is a 1-sphere $S$ of $\operatorname{Lk}(a, A)$ that is null homotopic in $(\mathrm{Lk}(a, M)-\mathrm{Lk}(a, A))$ $\cup S$. Thus there is a 3-cell $C$ in $\operatorname{St}(a, M)$ with $A \cap C=A \cap B d C=a^{*} S$. Let $B=\mathrm{Cl}(\mathrm{Bd} C-a * S) . B$ is a 2 -cell. A $B$-move expands $A$ to $A \cup C$ and collapses onto $(A-a * S) \cup a * y \cup B$ where $y$ is a point of $S$.

Move C: Let $a$ be a $(0, q)$-point of $A$ with $q>2$. Suppose that $x$ is a point of $\operatorname{Lk}(a, A)$ and $a * x$ may be extended to an $\operatorname{arc} B$ in $A$ with $\operatorname{Bd} B=a \cup y$, where $y$ is a $(p, q)$-point of $A$ with $p q \neq 0$, such that Int $B$ contains only $(0,2)$-points. Let $z \neq x$ be a point of $\operatorname{Lk}(a, A)$. There is a 2-cell $C$ in $M$ with $A \cap C=A \cap \mathrm{Bd} C=B \cup a * z$. Let $D$ $=\mathrm{Cl}(\mathrm{Bd} C-(a * z \cup B))$. A $C$-move expands $A$ to $A \cup C$ and collapses from $a * z$ across $C$ onto $(A-a * z) \cup a \cup D$.

Move D: Let $a$ be a $(1,1)$-point of $A$ with $x$ an isolated point of $\mathrm{Lk}(a, A)$. Suppose that $y$ is a $(p, q)$-point and that there is an arc $C$ in $A$ with Bd $C=a \cup y$ and Int $C$ containing only $(1,0)$-points. Then there is a 2-cell $B$ in $M$ with $A \cap B=A \cap \mathrm{Bd} B=C \cup a * x$. Let $D$ $=\mathrm{Cl}(\mathrm{Bd} B-(C \cup a * x))$. A $D$-move expands $A$ to $A \cup B$ and collapses from $a * x$ across $B$ onto $(A-a * x) \cup a \cup D$.

Move E: Let $a$ be a $(1,1)$-point of $A$ with $x$ the point and $S$ the 1 -sphere of $\mathrm{Lk}(a, A)$. Suppose that $a * x$ may be extended to an arc $B$ in $A$ with $\mathrm{Bd} B=a \cup y$, where $y$ is a $(p, q)$-point with $p q \neq 0$, and Int $B$ containing only $(0,2)$-points. There is a 3-cell $C$ of $M$ with $A \cap C$ $=B \cup a * S$ and $A \cap B d C=y \cup a * S$, such that $C$ collapses onto $B \cup a * S$. Let $D=\mathrm{Cl}(\mathrm{Bd} C-a * S)$. An $E$-move expands $A$ to $A \cup C$ and collapses from $a * S$ across $C$ onto $(A-(B \cup a * S)) \cup D$.

We observe that each move transforms $A$ into a spine of $M$. By a finite series of $A$-moves we may assume that each vertex of $A$ is either a $(1,1)$-point, a $(0, q)$-point or a $(p, 0)$-point. By $A$ - and $B$-moves we may assume that each $(p, 0)$-point of $A$ has $p=1$. By $A$ - and $C$-moves each vertex is a $(1,1)-,(1,0)-$, or $(0,2)$-point. By $D$ - and $E$-moves we obtain:

LEMMA 2. If $M$ has a spine that contains no wild arcs, $M$ has a spine that is the one-point union of 1-spheres and closed 2-manifolds. 
By Lemma 2, we may assume that $A$ is the one point union of $n$ 1-spheres $S_{1}, \cdots, S_{n}, k$ orientable 2 -manifolds $T_{1}, \cdots, T_{k}$, and $m$ nonorientable 2-manifolds $P_{1}, \cdots, P_{m}$.

LEMMA 3. If $P$ and $Q$ are homeomorphic 2-manifolds embedded in orientable 3-manifolds $M$ and $N$ respectively, $N(P, M)=N(Q, N)$, where $N(P, M)$ is the second derived neighborhood of $P$ in $M$.

Proof. Triangulate $M$ and $N$ so that $P$ and $Q$ are simplicially homeomorphic under the induced triangulation. Expand $P$ by tubing up its 1-skeleton and collapse the 3-ball neighborhood of each vertex onto a house with two rooms. Then push the walls of the house along the tubes. By collapsing carefully we transform $P$ into a standard spine $P^{\prime}$ of $N(P, M)$. We do the same with $Q$ obtaining a standard spine $Q^{\prime}$ of $N(Q, N)$. By collapsing carefully we may assume that $P^{\prime}$ and $Q^{\prime}$ are simplicially homeomorphic. By Corollary 1 of [1], the result follows.

Lemma 4. Each $T_{i}$ is a 2-sphere and each $P_{i}$ is a real projective plane.

Proof. Since $N(A, M)=\left(\bigcup_{i=1}^{n} N\left(S_{i}, M\right)\right) \cup\left(\bigcup_{i=1}^{k} N\left(T_{i}, M\right)\right)$ $\cup\left(\bigcup_{i=1}^{m} N\left(P_{i}, M\right)\right)$ has a 2-sphere boundary, $\mathrm{Bd} N\left(T_{i}, M\right)$ less two disks embeds in $S^{2}$. Thus $\mathrm{Bd} N\left(T_{i}, M\right)$ is one or two 2-spheres. By the same argument $\mathrm{Bd} N\left(P_{i}, M\right)$ is one or two 2-spheres. Suppose that $M$ is orientable. Since $T_{i}$ embeds in $R^{3}, N\left(T_{i}, R^{3}\right), N\left(T_{i}, M\right)$ and $T_{i} \times I$ are homeomorphic. Thus $T_{i}$ is a 2 -sphere if $M$ is orientable. By the local properties of the second derived neighborhood, if $P_{i}$ has genus greater than $2, \mathrm{Bd} N\left(P_{i}, M\right)$ contains a torus with a disk removed. Since the Klein bottle embeds in $S^{1} \times S^{2}$ and has a regular neighborhood with a torus boundary, $P_{i}$ is not a Klein bottle. Thus if $M$ is orientable $P_{i}$ is a real projective plane.

To complete the proof we need only show that $N\left(T_{i}, M\right)$ and $N\left(P_{i}, M\right)$ are orientable. Let $N$ denote either $N\left(T_{i}, M\right)$ or $N\left(P_{i}, M\right)$. Suppose that $N$ is not orientable. If $\mathrm{Bd} N$ is one 2-sphere, attach a 3-ball $E$ to $N$. From the $M-V$ sequence of $N$ and $E$ we obtain:

$$
0 \rightarrow Z \rightarrow \mathrm{H}_{2}(\mathrm{~N}) \rightarrow \mathrm{H}_{2}(\mathrm{~N} \cup E) \rightarrow 0 .
$$

This discounts $N=N\left(P_{i}, M\right)$. If $T_{i}$ has genus $g, \chi(N \cup E)=1-2 g=0$, where $\chi$ denotes the Euler characteristic. This contradiction implies that $\mathrm{Bd} N$ is two 2-spheres. Let $E$ and $F$ be two 3-balls attached to $N$. From the $M-V$ sequence of $N$ and $E \cup F$ we obtain:

$$
0 \rightarrow Z \oplus Z \rightarrow \mathrm{H}_{2}(\mathrm{~N}) \rightarrow \mathrm{H}_{2}(\mathrm{~N} \cup \mathrm{E} \cup F) \rightarrow \cdots
$$

This contradiction implies that $N$ is orientable and so the lemma is established. 
Since each 2-manifold is either $S^{2}$ or $R P^{2}$, Poincare duality and the universal coefficient theorem implies that $k=n$.

Let $L=P_{1} \cap \mathrm{Lk}(a, M)$. If $L$ is nullhomotopic in $\mathrm{Lk}(a, M)-A$, we may change $A$ by a $B$-move into $\mathrm{Cl}\left(A-P_{1}\right) \cup B \cup P^{\prime}$, where $B$ is a straight line segment from $a$ to a point of $L$ and $P^{\prime}$ is obtained from $P_{1}$ by radically projecting $L * a$ onto a disk of $\mathrm{Lk}(a, M)$ bounded by $L$. If we change $A$ in this way, we will say that $P_{1}$ is put on a stick. If $L$ is not nullhomotopic in $\operatorname{Lk}(a, M)-A$, let the two disks of $\operatorname{Lk}(a, M)$ bounded by $L$ be $D$ and $D^{\prime}$. Since $P_{1}$ is a projective plane, consider $P_{1}$ as a disk $E$ with a Moebius band $F$ attached. Let $F^{*}$ be the central curve of $F$ and let $E^{*}$ be an arc in $E \cup F$ from $a$ to a point of $F^{*}$. By a series of elementary moves, push the arcs and disks of $(A \cap a * D)$ $-P_{1}$ from $a$ along $E^{*}$, once around $F^{*}$ and back to $a$ along $E^{*}$ keeping $A-\operatorname{Int}(a * D)$ fixed. Thus, since $P_{1}$ is one-sided in $M$, we may assume that $L=P_{1} \cap \operatorname{Lk}(a, M)$ is nullhomotopic in $(\mathrm{Lk}(a, M)-A) \cup L$. Thus we have established:

Lemma 5. $P_{1}$ may be put on a stick.

Since $\operatorname{Bd} N(A, M)$ is connected, $T_{1}$ cannot be put on a stick. However we can show that:

Lemma 6. If $m=0, A$ may be picked so that $S_{1} \bigvee T_{1}$ may be put on a stick.

Proof. We define the following move:

Move F: Let $a$ be the join point of $A$. Suppose that $n>1$ and $x_{1}$ and $x_{2}$ are isolated points of $\operatorname{Lk}(a, A)$ that lie in the same component of $\operatorname{Lk}(a, M)-\left(\bigcup_{i=1}^{n} T_{i}\right)$. Since $\operatorname{Bd~} N(A, M)$ is connected $x_{1}$ and $x_{2}$ belong to distinct 1 -spheres of $A$, say $S_{1}$ and $S_{2}$. There is a 2-ball $B$ in $M$ with $A \cap B=A \cap \operatorname{Bd} B=a * x_{1} \cup a * x_{2}$. Let $C=\mathrm{Cl}\left(\mathrm{Bd} B-\left(a * x_{1} \cup a * x_{2}\right)\right)$. There is a 2 -ball $D$ in $M$ with $A \cap D$ $=A \cap \mathrm{Bd} D=\mathrm{Cl}\left(S_{2}-a * x_{2}\right) \cup x_{1}$ and $B \cap D=B \cap \mathrm{Bd} D=C$. Let $E$ $=\mathrm{Cl}\left(\mathrm{Bd} D-\left[\mathrm{Cl}\left(S_{2}-a * x_{2}\right) \cup C\right]\right)$. An $F$-move expands $A$ to $A \cup B \cup D$ and collapses from $a * x_{1}$ across $B \cup D$ onto $\left(A-a * x_{1}\right) \cup a \cup E$.

Since $m=0$, there is a 2 -sphere, say $T_{1}$, such that $L=T_{1} \cap \mathrm{Lk}(a, M)$ bounds a disk in $\operatorname{Lk}(a, M)-\cup_{i=2}^{n} T_{i}=X$. Let $E$ be the other component of $X$ bounded by $L$. By a series of $F$-moves we may assume that each $S_{i}$ meets $E$ and that $S_{1}$ meets $D$. By elementary moves the lemma is established.

By Lemma 5, $N(A, M)$ is homeomorphic with $N\left(\mathrm{Cl}\left(A-P_{1}\right), M\right)$ $\triangle N\left(P^{\prime}, M\right)$. By attaching a 3-ball to $N(A, M)$ it follows that $M$ $=M^{\prime} \# M^{\prime \prime}$, where $M^{\prime}$ is obtained by attaching a 3 -ball to $N\left(\mathrm{Cl}\left(A-P_{1}\right), M\right)$ and $M^{\prime \prime}$ is obtained by attaching a 3-ball to $N\left(P^{\prime}, M\right)$. Since $P^{\prime}$ is a spine of $M^{\prime \prime}$, Lemma 3 the uniqueness of the 
regular neighborhood up to isotopy, imply that $M^{\prime \prime}$ is topologically $R P^{3}$. By the obvious inductions $M=M^{\prime} \# M^{\prime \prime}$, where $M^{\prime \prime}$ is the connected sum of $m$ copies of $R P^{3}$ and $M^{\prime}$ is obtained by attaching a 3-ball to $N\left(\mathrm{Cl}\left(A-\bigcup_{i=1}^{m} P_{i}\right)\right)$. By Lemma $6, N\left(\mathrm{Cl}\left(A-\bigcup_{i=1}^{n} P_{i}\right), M^{\prime}\right)$ $=N\left(S_{1} \bigvee T_{1}, M^{\prime}\right) \triangle N\left(\mathrm{Cl}\left[\mathrm{Cl}\left(A-\cup_{i=1}^{n} P_{i}\right)-S_{1} \bigvee T_{1}\right], M^{\prime}\right)$ and so $M^{\prime}$ $=M_{1} \# M^{*}$, where $M_{1}$ and $M^{*}$ are obtained by attaching 3-balls to the first and second terms of the disk sum respectively. By induction $M^{\prime}=M_{1} \# \ldots \# M_{n}$, where each $M_{i}$ has $S_{i} \bigvee T_{i}$ as a spine. Since $N\left(S_{i}, M_{i}\right)$ is either a solid torus or a solid Klein bottle, $N\left(S_{i}, M_{i}\right) \cup T_{i}$ collapses onto a standard spine of either $S^{1} \times S^{2}$ less an open 3-ball or $J$ less an open 3-ball. Thus $M_{i}=S^{1} \times S^{2}$ or $J$.

Therefore the theorem is established.

\section{REFERENCES}

1. B. G. Casler, An imbedding theorem for connected 3-manifolds with boundary, Proc. Amer. Math. Soc. 16 (1965), 559-566.

2. P. H. Doyle, Two weak Poincare theorems, Proc. Cambridge Philos. Soc. 62 (1966), 23.

3. E. E. Moise, Afine structures in 3-manifolds. V: The triangulation theorem and Hauptvermutung, Ann. of Math. (2) 56 (1952), 96-114.

4. C. A. Persinger, Subsets of n-books in E3, Pacific J. Math. 18 (1966), 169-173.

Michigan State University and

The UNIVERSITY OF Nebraska 